\title{
PERCEPÇÃO DAS MERENDEIRAS DE CARAPICUÍBA - SÃO PAULO, BRASIL, SOBRE ASPECTOS DA SUA ATUAÇÃO PROFISSIONAL
}

\author{
Nyvian Alexandre Kutz ${ }^{1}$, Danielle Martins Pereira ${ }^{1}$, Marianne de Faria Chimello ${ }^{1}$, Alfredo \\ Almeida Pina-Oliveira ${ }^{2}$, Denise Cavallini Cyrillo³, Marcia Maria Hernandes de Abreu de Oliveira \\ Salgueiro ${ }^{1,4}$ \\ ${ }^{1,4}$ Centro Universitário Adventista de São Paulo, Brasil.nyvian.kutz@unasp.edu.br, d.pmartins@outlook.com \\ marianne-fc@hotmail.com,marciasalgueironutricionista@yahoo.com.br, marciasalgueironutricionista@yahoo.com.br \\ 2Universidade de São Paulo, Brasil. dccyrill@usp.br \\ 33niversidade Universus Veritas Guarulhos (UNIVERITAS-UNG), Brasil, aapo_enf@yahoo.com.br
}

\begin{abstract}
Resumo. Introdução: A promoção de uma alimentação adequada no ambiente escolar tem como personagem fundamental as merendeiras, consideradas agentes de saúde na escola, que tendem a repassar seus hábitos alimentares aos alunos. Objetivo: Analisar a percepção de merendeiras sobre alimentação saudável e aspectos da sua atuação profissional. Método: Estudo transversal, qualitativo, desenvolvido com merendeiras das escolas municipais de Carapicuíba. Foi utilizada a técnica de entrevista semiestruturada individuais e a análise de conteúdo temática aplicada às transcrições para a compreensão crítica do assunto. Resultados: A idade média das 32 merendeiras entrevistadas era de 53,03 anos; classificadas em obesidade; a maioria vivendo com companheiro e pertencentes à classe socioeconômica C. Foram identificados oito temas emergentes das entrevistas: Alimentação em casa versus alimentação na escola; Alterar o cardápio; Trocas de experiências culinárias; Alimentação saudável; Alimentação escolar; Alimentação e a saúde dos alunos; Papel da merendeira e; Insegurança alimentar e nutricional; Conclusões As percepções sobre alimentação saudável apontam para um conhecimento do conceito do termo, mas os demais apontamentos demonstram dificuldade de aplicar o conceito na prática. As concepções sobre os diversos aspectos de sua atuação remontam à importância do seu papel na alimentação escolar, manifestado em cada tema encontrado.
\end{abstract}

Palavras-chave: Estado Nutricional; Alimentação Escolar; Hábitos Alimentares; Promoção da Saúde; Pesquisa Qualitativa.

PERCEPTION OF THE SCHOOL COOKS OF CARAPICUÍBA - SÃO PAULO, BRAZIL, ON ASPECTS OF THEIR PROFESSIONAL PERFORMANCE

Abstract. Introduction: The promotion of adequate food in the school environment has as its fundamental character the school cooks, considered health agents at school, who tend to pass on their eating habits to students. Goal: Analyze the perception of school cooks about healthy eating and aspects of their professional performance. Method: Cross-sectional, qualitative study, developed with school cooks from the municipal schools of Carapicuíba. The individual semistructured interview technique and thematic content analysis applied to the transcripts were used to critically understand the subject. Results: The average age of the 32 interviewed school cooks was 53.03 years; classified in obesity; the majority living with a partner and belonging to socioeconomic class C. Eight themes emerged from the interviews were identified: Food at home versus food at school; Change the menu; Exchanges of culinary experiences; Healthy eating; School feeding; Food and student health; Role of the school cooks and; Food and nutritional insecurity. Conclusions: The perceptions about healthy eating point to a knowledge of the concept of the term, but the other notes demonstrate difficulty in applying the concept in practice. The conceptions about the different aspects of its performance go back to the importance of its role in school feeding, manifested in each theme found.

Keywords: Nutritional Status; School Feeding; Eating Habits; Health Promotion; Qualitative Research. 


\section{INTRODUÇÃO}

A promoção da alimentação adequada no ambiente escolar envolve toda a comunidade escolar, mas tem as merendeiras como elemento operacional central, consideradas como agentes de saúde na escola (Xavier et al., 2011a).

A participação das merendeiras na escolha dos alimentos pode determinar mudanças no cardápio oferecido, com base na percepção das mesmas acerca das preferências alimentares e a aceitação dos alunos, ou por seu conhecimento sobre os estoques disponíveis nas escolas (Carvalho et al., 2008), visando minimizar desperdícios e a utilização racional dos gêneros.

A escola como local de promoção de saúde, por meio do Programa Nacional de Alimentação Escolar (PNAE), deverá fazer a introdução de novos alimentos (ABERC, 2008), em quantidade e qualidade adequados a cada fase do desenvolvimento (Brasil, 2012), a partir de um cardápio adequado, elaborado pelo nutricionista responsável técnico, consultando os outros atores envolvidos na alimentação escolar, como merendeiras, gestores e os próprios alunos (Brasil, 2013).

Os hábitos alimentares se desenvolvem ao longo da vida, de forma dinâmica, e podem mudar durante o crescimento. Assim, hábitos alimentares saudáveis devem ser estimulados precocemente, pois o aprendizado adquirido na infância e adolescência permanece durante todo o ciclo vital (Madruga et al., 2012).

As merendeiras, envolvidas diretamente na alimentação escolar, conseguem transformar as regras e leis que o Governo Federal estabelece, em refeições saborosas. Seu papel é essencial para o sucesso das políticas públicas de Educação Alimentar e Nutricional (EAN) e Segurança Alimentar e Nutricional (SAN) (Brasil, 2018). A alimentação escolar é preparada por estes profissionais que, indiretamente, tendem a repassar seus hábitos alimentares aos alunos (Xavier et al., 2011b) influenciando o consumo alimentar dos mesmos (Accioly, 2009).

Também possui os recursos humanos necessários: nutricionistas responsáveis pelo PNAE; gestores e professores que devem incluir o tema alimentação saudável e adequada no plano pedagógico (Brasil, 2013); merendeiras capacitadas para fornecer refeições nutricionalmente equilibradas; pais que atuam ativamente no Conselho de Alimentação 
Escolar e; alunos que participam no aperfeiçoamento das práticas alimentares exercidas na escola (Brasil, 2009).

Nesse contexto, cabe apresentar as percepções que as merendeiras têm sobre os princípios que norteiam uma alimentação saudável, como essa percepção reflete em seu cotidiano e, consequentemente, como se expressam em suas atividades profissionais; além da autopercepção de seu papel na educação nutricional do alunado.

Sendo assim, este trabalho se propõe a analisar a percepção de merendeiras sobre alimentação saudável e aspectos da sua atuação profissional.

\section{MÉTODO}

Foi um estudo transversal, qualitativo, desenvolvido com merendeiras de 44 escolas municipais de Carapicuíba - SP, divididas em 15 creches (berçário e maternal), 23 de educação infantil (pré I e pré II) e 6 de ensino fundamental anos iniciais. Foram identificadas 145 merendeiras que preparavam e serviam refeições e lanches conforme cardápio definido pelas nutricionistas vinculadas à Secretaria Municipal de Educação. A modalidade creche contava com $31,03 \%$ das merendeiras da rede municipal, a educação infantil com $52,41 \%$ e o ensino fundamental anos iniciais com $16,56 \%$.

Todas as merendeiras foram convidadas a participar da pesquisa por meio de convite realizado oralmente na reunião anual de capacitação, em julho de 2017.

O projeto foi aceito pela Secretaria Municipal de Educação de Carapicuíba e aprovado pelo Comitê de Ética em Pesquisa da Faculdade de Ciências Farmacêuticas da Universidade de São Paulo sob o CAAE 69597617.0.0000.0067. Após a devolutiva dos Termos de Consentimento Livre e Esclarecido assinados, iniciou-se a coleta de dados.

Vale destacar que este manuscrito apresenta uma parte dos dados coletados na pesquisa original, que contou com a participação de 68 merendeiras para a coleta de dados quantitativos (caracterização, classificação socioeconômica, antropometria, perfil do consumo alimentar e outros hábitos de vida e, qualidade de vida), das quais 32 foram selecionadas para a coleta de dados qualitativa. Para tanto, foi utilizada amostragem por conveniência, considerando a modalidade da escola (creche, educação infantil e ensino fundamental anos iniciais) e sua representatividade dentro da amostra. 
As percepções das merendeiras foram obtidas por meio de entrevista semiestruturada, pois segundo Minayo (2010), essa técnica é orientada por um roteiro, mas com abertura a outras questões que a aprofundam. Nesse caso, objetivou-se compreender a percepção das merendeiras sobre alimentação saudável e aspectos da sua atuação profissional, concepções da alimentação das participantes, da alimentação escolar, do próprio trabalho e de suas responsabilidades.

O roteiro de perguntas utilizado nesta pesquisa apresentava as seguintes perguntas: 1 . Como é a sua alimentação quando não está na escola? 2. Existem semelhanças e/ou diferenças entre as preparações que você faz na escola e em casa? Quais seriam? 3. Existe uma troca de experiência na formulação das preparações? 4. O que você entende por alimentação saudável? 5. Você considera a alimentação escolar como saudável? Se não, o que precisaria ser alterado para mudar esse quadro? 6. De que forma a merenda escolar influencia a saúde dos alunos? 7. Como você descreveria o seu papel como merendeira?

Este roteiro foi testado num piloto, sendo aplicado com duas merendeiras, que foram excluídas do levantamento final das entrevistas, a fim de avaliar o entendimento das questões e a qualidade das respostas obtidas. As entrevistas foram gravadas, em local reservado, individualmente, garantindo o sigilo das participantes.

Do total das 44 escolas, 14 apresentaram recusa de todas as merendeiras atuantes para participarem da pesquisa, por meio de carta assinada e enviada pela diretoria de cada escola em resposta à pesquisadora responsável.

Das 30 escolas participantes, participaram 32 merendeiras nas seguintes proporções: $25 \%$ na modalidade creche, $59,38 \%$ em educação infantil e 15,63\% no ensino fundamental anos iniciais.

Os discursos obtidos nas entrevistas foram transcritos e analisados utilizando-se para tal a Análise de Conteúdo Temática proposta por Bardin (2009) e Bernard, Wutich e Ryan (2017), para a compreensão crítica do assunto.

$\mathrm{Na}$ pré-análise foi realizada leitura flutuante de todas as entrevistas, que constituíram o corpus do estudo, objetivando-se definir os temas, utilizando como indicador a repetição e relevância. Tanto a construção dos temas quanto as hipóteses foram realizadas a posteriori, de forma indutiva. 
Optou-se por utilizar abordagem êmica, cuja visão dos observados, de forma interna, adotam uma postura particular, única e analítica (Rosa \& Orey, 2012).

$\mathrm{Na}$ etapa exploratória do material utilizou-se a técnica manipulativa de cutting and sorting (Ryan \& Bernard, 2003), que envolve a identificação das citações ou expressões que de alguma forma parecem importantes e depois organização delas em seções de sentido comum, agrupando os trechos. Foi tomado o cuidado, ao grifar o material, de manter o sentido/contexto de modo que, após realçar, o trecho permanecesse inteligível. Não foi estabelecida uma unidade de registro (número de palavras ou quantidade de linhas), utilizando-se apenas a unidade de contexto, definida como frase (Bardin, 2009).

Os trechos foram agrupados, por cores, de acordo com a repetição de ideias e pelas semelhanças nos sentidos. Em seguida, analisaram-se os discursos a fim de formar as categorias de análise (temas) (Bardin, 2009).

Após essas etapas, iniciou-se a análise, primeiramente por meio da construção de um codebook (guia de codificação), a fim de apresentar os temas, suas definições e como deveriam ser aplicados (Bardin, 2009; Macqueen, Mclellan \& Milstein, 1998; Bernard, Wutich \& Ryan, 2017) e sua aplicação pela pesquisadora responsável.

A segunda etapa da análise se deu pela aplicação do codebook por uma segunda codificadora, com auxílio dos softwares Word e Excel, ambos do pacote Microsoft Office para Windows, ano 2013, dando a liberdade para criar novos temas e/ou agrupar temas existentes. A pesquisadora convidada para a codificação foi a coorientadora desta pesquisa, por ter contato com a pesquisa e estar familiarizada com o tema, a metodologia e a aplicação do codebook.

Em seguida, avaliou-se a concordância entre as codificadoras quanto às categorizações, discutindo cada tema até obter um consenso. Feito isso, somou-se, para cada tema: (1) o número de unidades de análise que as codificadoras categorizaram de maneira igual, (2) o número de unidades de análise que somente a codificadora 1 categorizou, (3) o número de unidades de análise que somente a codificadora 2 categorizou e, após discussão para consenso, (4) o número de unidades de análise que não foram categorizadas. Desta forma, tornou-se possível o cálculo do coeficiente Kappa de Cohen (Bernard, Wutich \& Ryan, 2017), que foi calculado pelo software online GraphPad Quick-Calcs, e classificado pelo coeficiente de Landis e Koch (1977). 
As unidades de análise não categorizadas foram excluídas após acordo entre as codificadoras, que consideraram a relevância dos trechos para o tema proposto, trechos sobre experiências vividas por outras pessoas e trechos com sentido dúbio ou ambíguo, concluindo assim, a etapa de aplicação do codebook.

Os temas encontrados e seus nemônicos serão descritos na seção seguinte e apresentados segundo seus aspectos centrais e periféricos, ou seja, os aspectos que mais representam o tema, mais apareceram nas falas dos entrevistados e os que surgiram com menor frequência, de forma a extrair as principais informações obtidas pelas falas analisadas no codebook (Bernard, Wutich \& Ryan, 2017).

\section{RESULTADOS E DISCUSSÃO}

A idade média das 32 merendeiras estudadas era de 53,03 anos; com circunferência da cintura média de $96,3 \mathrm{~cm}$, indicativo de risco substancialmente elevado para doença metabólica; média de $30,36 \mathrm{Kg} / \mathrm{m}^{2}$ de índice de massa corporal, classificando obesidade; $59,38 \%$ vivendo com companheiro e $62,5 \%$ pertencentes à classe socioeconômica $C$.

A análise de conteúdo temática das entrevistas permitiu identificar oito temas emergentes, sendo eles Alimentação em casa versus alimentação na escola (ACE), Alterar o cardápio (ALT), Trocas de experiências culinárias (TEC), Alimentação saudável (ALS), Alimentação escolar (AES), Alimentação e a saúde dos alunos (ASA), Papel da merendeira (PAM) e Insegurança alimentar e nutricional (IAN). A confiabilidade calculada pelo coeficiente Kappa variou entre substancial e quase perfeita.

O primeiro tema tem enfoque na alimentação da própria merendeira e traz nuances da alimentação em casa versus alimentação na escola. Este tema traz as diferentes formas de cozinhar no contexto familiar e para os alunos. Tem como aspecto central o uso de temperos diferenciados quando se cozinha em casa, indicando que em casa elas utilizam temperos naturais e industrializados, atendendo às preferências da família. $\mathrm{Na}$ escola utilizam os temperos disponibilizados pela Prefeitura, definidos pelas nutricionistas responsáveis pela alimentação escolar, que são exclusivamente temperos frescos e naturais (alho, cebola, coentro, salsinha e cebolinha).

Neste tema, observaram-se dois aspectos periféricos, sendo um o cardápio da escola, que impede uma variedade maior de preparações, ao passo que em casa existe maior liberdade. 
O outro aspecto corresponde à utilização de gordura em maior quantidade quando se cozinha em casa.

O segundo tema diz respeito a alterar o cardápio, que apresenta casos em que a merendeira precisa alterar o cardápio pela ocorrência de imprevistos para a execução do mesmo. Este tema apresenta um aspecto central baseado na alteração do modo de preparo, onde esclarecem não possuírem autonomia para modificar o cardápio a menos que alterem as preparações planejadas utilizando os mesmos ingredientes programados para o dia.

Apesar de apontarem não poder alterar o cardápio, elas conseguem criar "brechas" para modificar e incrementar preparações que melhorem tanto a aceitação quanto a qualidade nutricional do cardápio, agrupando saberes e percepções pessoais a conhecimentos técnicos, visando a promoção da saúde dos alunos.

O terceiro tema traz as trocas de experiências culinárias que as merendeiras fazem entre si, com a comunidade escolar e que levam ou trazem de casa. O aspecto central deste tema é a busca pela melhor aceitação do cardápio e ressalta-se que trocas de experiências culinárias realizadas por merendeiras de escolas diferentes, como é o caso da receita do peixe famosa em uma das escolas, favorece a aceitação desse tipo de alimento, tanto nas escolas quanto com as famílias das merendeiras.

Outro destaque é o bolo de chocolate, que a receita foi apresentada no cardápio escolar, pelas nutricionistas, foi testada em uma escola com boa aceitação pelos alunos e por isso foi compartilhada não só entre as merendeiras, mas também com a comunidade escolar e seu entorno.

Isso reforça que trocas de experiências culinárias facilitam o serviço, promovem melhorias nos cardápios e minimizam desperdícios. Esse processo educativo aproxima todos os atores envolvidos na alimentação escolar na busca de um cardápio saudável e atrativo para os alunos.

O quarto tema traz os conceitos sobre alimentação saudável na percepção das merendeiras. As frutas, verduras e legumes aparecem como aspecto central do tema, juntamente a outros grupos alimentares como cereais, leguminosas e carnes. 
As falas sobre alimentação saudável das merendeiras estão de acordo com as recomendações do Guia Alimentar para a População Brasileira (Brasil, 2014), que valoriza o consumo de alimentos frescos, minimamente processados, descascando mais e desembalando menos. Esses conhecimentos demonstram que as profissionais já incorporaram a temática alimentação saudável, refletindo os investimentos públicos no Município. Embora as merendeiras destaquem os grupos alimentares de açúcares e doces; óleos e gorduras; feijões; carnes e ovos; frutas; legumes e verduras e; cereais pães e massas (Brasil, 2008), o grupo do leite e seus derivados não aparece no conceito de alimentação saudável manifestado por elas.

Com base no tema anterior, o quinto tema traz a classificação da alimentação escolar em adequada e inadequada, dando continuidade ao que elas consideravam ser uma alimentação saudável, avaliando os gêneros recebidos e a alimentação preparada na escola. Por outro lado, o sexto tema ilustra a relação entre a alimentação e a saúde dos alunos. O aspecto central deste tema foi o estado nutricional, evidenciando que a alimentação escolar traz benefícios, principalmente para os alunos "magrinhos" com o passar do tempo.

O sétimo tema surge do papel da merendeira dentro da escola, em um sentido mais amplo que somente o preparo de refeições. O aspecto central aponta para o papel de educadora que desenvolvem, nem sempre reconhecido, mas que é exercido com muito respeito, no campo da alimentação e nutrição bem como da moral e da ética.

As ações de EAN realizadas pelas merendeiras em conjunto com a comunidade escolar nas escolas de Carapicuíba alinham-se às considerações da Resolução FNDE № 26 de 17 de junho de 2013, no que tange às especificações do Capítulo V do documento, "Das ações de educação alimentar e nutricional e de segurança alimentar e nutricional” (Brasil, 2013).

A apropriação de conhecimentos as permite construir novas formas de olhar aqueles a quem servem, de maneiras respeitosas e criativas, valorizando cada indivíduo e sua individualidade. Destaca-se a utilização do alimento como recurso educacional com foco na realização de reeducação alimentar com os alunos e o envolvimento de atores estratégicos nas ações de prática contínua e permanente, transdisciplinar, intersetorial e multiprofissional (Brasil, 2013). Evidencia-se também o papel de responsabilidade com a saúde dos alunos, em relação à higiene e no cuidado com os alunos com necessidades alimentares especiais. 
Por fim, o oitavo tema faz referência à insegurança alimentar e nutricional de alguns alunos, observada pelas merendeiras. Baseando-se no conceito de SAN apresentado no capítulo de revisão de literatura, indicadores relacionados ao acesso, consumo, das condições sociais, econômicas e de estado nutricional são utilizados para caracterização de situações de violação desse direito, ou seja, da insegurança alimentar e nutricional (André et al., 2018).

O aspecto central do tema demonstra que, entre os indicadores, a falta de acesso aos alimentos pelas famílias, com variedade como a da alimentação escolar, é o que mais se percebe entre os alunos. Outras situações de violação do direito também foram citadas pelas merendeiras como sendo indicadores de insegurança alimentar e nutricional, apresentados aqui como aspecto periférico do tema. $O$ estado nutricional dos alunos observado pelas merendeiras, o contexto e a estrutura familiar são percebidos pelas merendeiras.

Em suma, as percepções das merendeiras obtidas neste trabalho, observa-se a realização da SAN durante a execução das diretrizes recomendadas pelo PNAE, de forma natural e espontânea no preparo e fornecimento da alimentação escolar. Embora não apresentem em suas falas terminologias técnicas a respeito desse assunto, suas práticas, aliadas a saberes populares e técnicos, são indispensáveis para a concretização do direito humano à alimentação adequada.

Vale ressaltar que, ao observar o somatório de todos os resultados, encontram-se contradições entre os achados quantitativos e qualitativos. Pontua-se, por exemplo, as diferenças entre o que consideram saudável (teoria) e o que consomem (prática).

É possível observar que, na percepção delas, a alimentação escolar é por vezes o fator determinante para a permanência do aluno na escola, deixando o ensino em segundo plano, o que sinaliza o caráter assistencial da alimentação escolar.

Este estudo é pioneiro no Município, que não apresentava até então nenhuma pesquisa com este público. Como dito anteriormente, o PNAE é uma das políticas públicas mais antigas de SAN no país, mas poucos estudos que envolvam os atores responsáveis por sua execução estão disponíveis na literatura. 
Como limitações do estudo, aponta-se a não devolutiva das falas transcritas a cada participante e a escolha de utilização de um método tradicional para validar os resultados.

Vale ressaltar que, ao observar o somatório de todos os resultados da pesquisa, encontramos contradições entre os achados quantitativos e qualitativos. Pontua-se, por exemplo, as diferenças entre o que consideram saudável (teoria) e o que consomem (prática), onde a teoria esstá alinhada com as diretrizes estabelecidas e adotadas pelas instituições de ensino, porém a prática cotidiana apresenta pontos importantes em desconsonância, reafirmando que conhecimento não garante a adoção de hábitos alimentares saudáveis.

\section{CONCLUSÕES}

As percepções sobre alimentação saudável apontam para um claro conhecimento do conceito do termo, mas os demais apontamentos demonstram a dificuldade de se aplicar o conceito na prática. As diferenças apontadas na alimentação que elas realizam em casa e na escola são baseadas naquilo que é melhor para os alunos, como o tempero natural, pouco sal e a ausência de frituras na escola. Elas reconhecem que não se deve alterar o cardápio a menos que falte algum gênero alimentício ou que seja necessário incrementar alguma preparação. Percebem que as trocas de experiências culinárias melhoram a aceitação do cardápio. Destacam que é visível a influência da alimentação escolar sobre a saúde dos alunos, principalmente com melhorias no estado nutricional e no aprendizado discente. Afirmam que seu papel na alimentação escolar extrapola seu preparo e fornecimento de refeições saudáveis e nutricionalmente adequadas, pois se identificam como educadoras no âmbito da formação de hábitos alimentares saudáveis. Reconhecem que se trata de uma profissão requer grande responsabilidade, principalmente com os alunos que têm necessidades alimentares especiais e com aqueles que se encontram em insegurança alimentar e nutricional. Os achados deste estudo demonstram que o uso de metodologias qualitativas enriquece e amplia a compreensão que se tem do objeto de estudo. A triangulaçao de dados é útil e imprescindível para uma formulação de pensamento mais aprofundada a respeito daquilo que se pesquisa.

\section{REFERÊNCIAS}

ABERC. Associação Brasileira das Empresas de Refeições Coletivas. (2008). Anais do IV Fórum Nacional de Merenda Escolar.

Accioly, E. (2009). A escola como promotora da alimentação saudável. Ciência em tela, 2(2), 01-09. Recuperado de http://www.cienciaemtela.nutes.ufrj.br/artigos/0209accioly.pdf. 
André, H. P., Sperandio, N., Siqueira, R. L., Franceschini, S. C. C., Priore, S. E. (2018). Indicadores de insegurança alimentar e nutricional associados à anemia ferropriva em crianças brasileiras: uma revisão sistemática. Ciência e Saúde Coletiva, 23(4), 1159-1167. Recuperado de https://doi.org/10.1590/141381232018234.16012016.

Bardin, L. (2009). Análise de conteúdo. (5th ed). Lisboa: Editora Edições 70.

Bernard, H. R., Wutich, A., Ryan, G. W. (2017). Analyzing qualitative data: systematic approaches. (2nd ed). Thousand Oaks, California: Sage.

Brasil. Fundo Nacional de Desenvolvimento da Educação. Ministério da Educação. Acesso à informação. Notícias. (2018b). Merendeiras brasileiras participam de intercâmbio cultural na República Dominicana. Recuperado de http://www.fnde.gov.br/acesso-a-informacao/institucional/area-deimprensa/noticias/item/11867-merendeiras-brasileiras-participam-de-interc\%C3\%A2mbio-cultural-narep\%C3\%BAblica-dominicana>. Acesso em: 03 jul. 2018.

Brasil. Ministério da Educação. Fundo Nacional de Desenvolvimento da Educação. (2013). Resolução no 26, de 17 de junho de 2013. Dispõe sobre o atendimento da alimentação escolar aos alunos da educação básica no âmbito do Programa Nacional de Alimentação Escolar. Brasília: Diário Oficial da União. Recuperado de http://www.fnde.gov.br/acesso-a-informacao/institucional/legislacao/item/4620-resolu\%C3\%A7\%C3\%A3ocd-fnde-n\%C2\%BA-26,-de-17-de-junho-de-2013>. Acesso em: 28 de mar. 2016.

Brasil. Ministério da Educação. Fundo Nacional de Desenvolvimento da Educação. Resolução no 38 , de 16 de julho de 2009. Dispõe sobre o atendimento da alimentação escolar aos alunos da educação básica no âmbito do Programa Nacional de Alimentação Escolar. Brasília: Diário Oficial da União, 2009. Recuperado de: $\quad$ http://www.fnde.gov.br/acesso-a-informacao/institucional/legislacao/item/3341 resolu\%C3\%A7\%C3\%A3o-cd-fnde-n\%C2\%BA-38-de-16-de-julho-de-2009>. Acesso em: 28 de jun. 2018.

Brasil. Ministério da Saúde. Secretaria de Atenção à Saúde. Departamento de Atenção Básica. (2012a). Política Nacional de Alimentação e Nutrição. Brasília: Ministério da Saúde. Recuperado de: http://dab.saude.gov.br/portaldab/biblioteca.php?conteudo=publicacoes/pnan>. Acesso em: $27 \mathrm{de}$ abr. 2017.

Brasil. Ministério da Saúde. Secretaria de Atenção à Saúde. (2008). Guia alimentar para a população brasileira: promovendo a alimentação saudável. Brasília: Ministério da Saúde. Recuperado de http://bvsms.saude.gov.br/bvs/publicacoes/guia_alimentar_populacao_brasileira_2008.pdf>. 16 out. 2016.

Brasil. Ministério do Desenvolvimento Social e Combate à Fome. (2012). Marco de referência de educação alimentar e nutricional para as políticas públicas. - Brasília, DF: MDS; Secretaria Nacional de Segurança Alimentar e Nutricional. Recuperado de http://www.cfn.org.br/wpcontent/uploads/2017/03/marco_EAN.pdf>. Acesso em: 30 de abr. 2017.

Carvalho, A. T., Muniz, V. M., Gomes, J. F., Samico, I. (2008). School meals program in the municipality of João Pessoa - PB, Brazil: school meal cooks in focus. Interface: Comunicação, Saúde e Educação, 12(27), 823834. Recuperado de https://doi.org/10.1590/S1414-32832008000400012.

IBGE. Instituto Brasileiro de Geografia e Estatística. Estatísticas. (2018b). Número de municípios e estimativa da população para 2017. Rio de Janeiro: IBGE. Recuperado de: https://downloads.ibge.gov.br/downloads_estatisticas.htm>. Acesso em: 23 de jun. 2018.

Landis, J. R., Koch, G. G. (1977). The measurement of observer agreement for categorical data. Biometrics, 33(1), 159-74. Recuperado de https://doi.org/10.2307/2529310.

MacQueen, K. M., McLellan, E., Milstein, K. K. B. (1998). Codebook development for team-based qualitative analysis. Cultural Anthropology Methods, 10(2), 31-36. Recuperado de https://doi.org/10.1177/1525822X980100020301.

Madruga, S. W., Araújo, C. L. P., Bertoldi, A. D., Neutzling, M. B. (2012). Manutenção dos padrões alimentares da infância à adolescência. Revista de Saúde Pública, 46(2), 376-386. Recuperado de https://doi.org/10.1590/S0034-89102012005000016.

Minayo, M. C. S. (2010). O desafio do conhecimento: pesquisa qualitativa em saúde. (12th ed). São Paulo: Hucitec.

Rosa, M., Orey, D. C. (2012). O campo de pesquisa em etnomodelagem: as abordagens êmica, ética e dialética. Educacão e Pesquisa, São Paulo, 38(4), 865-879. Recuperado de https://doi.org/10.1590/S151797022012000400006

Ryan, G. W., Bernard, H. R. (2003). Techniques to Identify Themes. Field Methods, 15(1), 85-109. Recuperado de https://doi.org/10.1177/1525822X02239569.

Xavier, V. L., Pereira, J. A., Silva, M. A. A., Zloccowick; N. M., Arruda, H. A. S. (2011a). Perfil socioeconômico e hábitos alimentares de merendeiras de escolas públicas do Estado de Pernambuco. Nutrire, 36(suppl.), 340.

Xavier, V. L., Silva, M. A. A., Pereira, J. A., Zloccowick, N. M., Arruda, H. A. S. (2011b). Perfil nutricional de merendeiras de escolas públicas do Estado de Pernambuco. Nutrire, 36(suppl.), 340. 